\title{
SOURCE OF OUTER ZONE PROTONS *
}

GPO PRICE

\$

CFSTI PRICE(S) \$

Hard copy $(\mathrm{HC}$

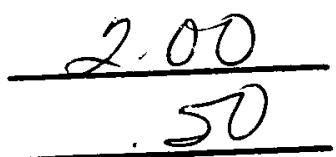

MSA TA: $: 55375$

Microfiche (MF)

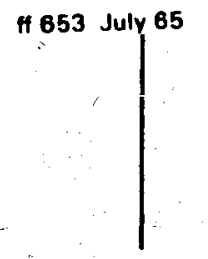

\section{SEPTEMBER 1965}

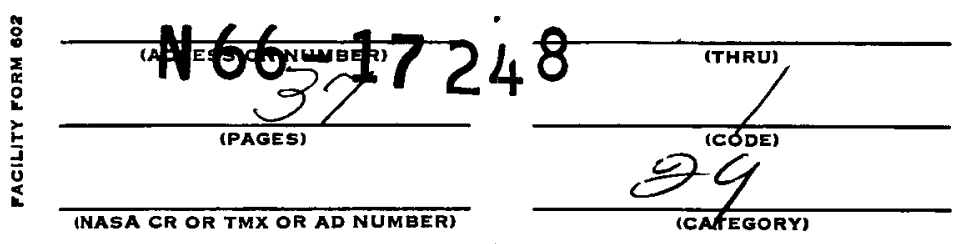

\section{NASA}

GODDARD SPACE FLIGHT CENTER GREENBELT, MARYLAND

\footnotetext{
* Presented at the Advanced Study Institute on Radiation Trapped in the Earth's Megnetic Field Bergen, Norway August 16 through September 3, 1965
} 
W. N. Hess

Introduction

In the $L=2$ to 5 range in the outer radiation belt, Davis, Hoffran, and Williamson [1964] find that the spectra of the relatively stable 0.1 to $5 \mathrm{Mev}$ protons show smooth but large variations with $L$ and equatorial pitch angle, $\alpha_{0}$, as in Fig. 1. Protons near the earth and at $\alpha_{0}$ near $90^{\circ}$ are more energetic than those at larger $L$ and at smaller $\alpha_{O}$. The spectra are well represented by $e^{-E / E_{0}}$.

Inspection of the experimental data showed that $E_{0} \propto \mathrm{L}^{-3} \propto \mathrm{B}$. This was very suggestive since to have a characteristic energy of the system vary with the magnetic field suggested betatron acceleration and suggested that the particles were moving radially across the field. Such a process must violate at least the third adiabatic invariant of motion since the particle moving radially aos not conserve the magnetic flux inside its orbit.

Kellogg [1959] first suggested that the radiation belt might be formed through magnetic disturbances in which the third adiabatic invariant of trapped particles is violated without violating the first and second invariants. Violation of the third invariant

* The first part of this paper follows closely the material in Nakada, Dungey and Hess, J.G.R., 70. 
allows motion in L-space. As particles move closer to the earth iney tend to gain energy with the maintenance of the first invariant since, for example, $E / B$ is a constant for $90^{\circ}$ pitch angles. So, this process can introduce acceleration of protons. Kellog 's suggestion has been adopted for this study although the mechanism for motion in $L$ space is unspecified. It has further been assumed that motion in L-space is rapid compared with atmospheric loss and scattering processes except very near the earth and that the geomagnetic field is sufficiently well represented by a dipole.

\section{Fiivergy and Angle Variations}

If the first and second adiabatic invariants of trapped particles are maintained during motion in I space, changes in both the energy and equatorial pitch angle can be calculated. The first invariant is

$$
\mu=\frac{E \sin ^{2} \alpha_{0}}{B_{0}}=\frac{E L^{3} \sin ^{2} \alpha_{0}}{.312}
$$

where $B_{0}$ is the equatorial magnetic field. The second invariant is

$$
J=m \oint v \cos \alpha d \ell=m v \overline{\cos \alpha} \ell=\sqrt{E} L F\left(\alpha_{0}\right)
$$


where $\mathrm{m}$ is the mass, $\mathrm{v}$ the velocity, $\alpha$ the local pitch angle and $\ell$ is along the guiding center. The integration is over a complete north-south oscillation.

Since $\mu$ and $J$ are constants, equation ( 1 ) may be divided by the square of equation (3) to give

$$
\text { L }\left[\frac{\sin \alpha_{0}}{F\left(\alpha_{0}\right)}\right]^{2}=\text { constant. }
$$

From this, the changes in $\alpha_{0}$ with $L$ have been found and are shown in Figure 2. As Davis and Chang [1962] have indicated, particles aiffusing inwards assume flatter helices and move closer to the equator.

These changes in $\alpha_{0}$ with $L$ and equation (1) may be used to find the variation in energy with $L$ and $\alpha_{O}$. Results are shown in rigure 3 for protons having $\alpha_{O}$ values at $L=7$ as indicated on the curves. Energies are reiative to eñergies at $L=7$.

The energy transformation is given by

$$
E L^{3} \sin ^{2} \alpha_{O L}=E_{S} L_{s}^{3} \sin ^{2} \alpha_{O S}
$$

where the subscript $s$ refers to the source. If the source spectrum has an exponential form, the transformation is given by 


$$
e^{-E_{S} / E_{O S}} \rightarrow e^{-\frac{E L^{3} \sin ^{2} \alpha_{O} L}{E_{O S} L_{S}{ }^{3} \sin 2 \alpha_{O S}}} \equiv e^{-E / E_{O}}
$$

which shows

$$
E_{O} I^{3} \sin ^{2} \alpha_{O L}=E_{O S} L_{S}^{3} \sin ^{2} \alpha_{O S}
$$

From this it can be seen that an exponential source remains exponential after $\mathrm{L}$ space motion and that $\mathrm{E}_{\mathrm{O}}$ changes in the same way with $L$ and $\alpha_{0}$ as has been calculated for a single particle in the previous section.

These two predictions of the model may be compared with experiment. The first prediction, that the spectra retains its exponential form, is in agreement with experiment. To test the second prediction, measured $E_{O}$ [Davis et al., 1964] have been plotted in Figure 4 as a function of $I$ with appropriate changes in $\alpha_{O}$ with $L$. The labels on the curves refer to $\alpha_{0}$ values at $\mathrm{L}=7$. The dashed curves in Figure 4 are taken from Figure 2 for corresponding changes in $E$ with $L$ and $\alpha_{O}$. The changes in $E_{O}$ with $L$ show good agreement between the model and experimental results. The experimental resuits also give the same trend as the model in the change in the siopes of the curves with $\alpha_{0}$. 
If tine dashed curves in Figure 4 are extended, they intersect near $I=i 0$. This intersection is where the spectrum is independent of $\alpha_{0}$ and thus gives a source location with the simplest assumptions aibout the source.

\section{Flux Variation with I}

Now let us consider the way the flux of particles will vary with motion in $L$. The density of particles in phase space, $f$, is given by

$$
f(v, \alpha)=\frac{d N}{d A d t p^{2} d p d \Omega}
$$

But, the pitch angle distribution in protons $/ \mathrm{cm}^{2}-\mathrm{sec}-\mathrm{ster}-\mathrm{Mev}$ measured by Davis, Hoffman and Williamson is

$$
j(E, \alpha)=\frac{d N}{d A d t d E d \Omega} .
$$

This gives

$$
f(v, \alpha)=\frac{j(E, \alpha) d E}{p^{2} v d p}
$$


or

$$
f(E, \alpha)=\frac{1}{2 \mathrm{mE}} j(E, \alpha)
$$

This shows the simple relationship between the measured fluxes and the velocity distribution function. Our model of the drift process has $\mu$ and $J$ constants of the motion. We can study the way the particle flux varies with position by studying

$$
f\left[E_{1}\left(\mu_{1}, J_{1}\right), \alpha_{1}\left(\mu_{1}, J_{1}\right), I_{1}\right]
$$

The values of $f$ were computed as functions of $L$ for the data of Davis and Williamson for many pairs of the values of $\mu$ and $J$, and Figure 5 shows $f$ plotted against $I$ for fixed $\mu$ and $J$. The curves for all $\mu$ and $J$ were nomalized in the region $L=4$ to compare their shapes and the following interesting empirical fact shows up

$$
f(\mu, J, I)=g(I) h(\mu, J)
$$


That is to say, the function $f$ is separable and the $L$ dependence is essentiaily the same for all $\mu$ and $I$. This being the case we can write

$$
\frac{f\left(\mu_{1}, J_{1}, L\right)}{f\left(\mu_{1}, J_{1}, 5\right)}=\frac{g(L)}{g(5)}=C(L)
$$

w...re $C^{\prime}(L)$ can be read directly from Fig. 5 and it can be used to transform $\therefore$ ixes from one $L$ to another. Fig. 5 shows $f$ varying considerably with $i$. It is seen that $(\partial f / \partial L)_{\mu J}$ is always positive, suggesting that the 1...icle source is at large $\mathrm{L}$, the particles duffusing inwards and loss frocesses reducing $f$ further in. The small slopes of Figure 5 at the Lurger I values implies that loss processes are probably relatively wininortant there, the slope probably being due to diffusion of particles away from the source at the outer boundary. The much larger slopes ai the lower $L$ values imply that loss processes are relatively important in this region.

We now have a scheme for transforming proton fluxes from one position iit 3,1 space to another position. Using equations (I) and (2) we k. W w how the energy $E_{0}$ of an exponential distribution varies and how the equatorial pitch angle changes. Using the data in Fig. 5 we have an empirically determined scheme for transforming particle fluxes as 
given by equation (12). Starting with the data of Davis, Hoffman and Williamson shown in Fig. 6 for equatorial particles at $L=5$, we have transformed this to $L=2.4$. This equatorial data has been fit by an expression

$$
j_{i}(E)=\sum_{i} a_{i} e^{-E / E} O i=3.8 \times 10^{7} e^{-E / .088}+1.65 \times 10^{5} e^{-E / .465}
$$

Obviously the second term in equation (13) is not very well known since from Fig. 6 there are only two points that can be used to fit this. This transformed data is compared with the Relay I data of Fillius and McIiwair in Fig. 7. Over the range where comparison is possible the agreement is very good. The transformed flux and the experimental flux ai $i \quad 2.4$ agree to within a factor of 2 which is as accurately as C(i); is known from Fig. 5. Beyond $E=18 \mathrm{Mev}$ the comparison is impossible. Davis' data runs out here. The experimentally measured fluxes at $I=2.4$ fall significantly above the extrapolation of Davis' data shown dotted in Fig. 7 suggesting that a third term should be added to the flux description in equation (13). For part of the following discussion we have added a third exponential

$$
j_{3}(E)=1.0 \times 10^{5} \mathrm{e}^{-E / 4.95}
$$


Off-Equator Protons

Having found reasonable agreement between measured fluxes and transformed fluxes for equatorial particles, let us now examine offequator particles having $\alpha_{0} \neq 90^{\circ}$. We start with a fit to the Davis, Hoffman and Williamson data at $L=5$ similar to that given in equation (13) but with values of the constants $a_{i}\left(\alpha_{0}\right)$ and $E_{0 i}\left(\alpha_{0}\right)$ varying with the pitch angle. Davis has made such a fit to his data so this initial data is well known. Using the same transformation as before involving equations (1), (2) and (12) the flux measured at $I=5$ has been transformed to other I values and is compared with the Relay I data $O \vec{i}$ Fillius and McIlwain for off-equator locations in Figures 8 to 2. In these figures the transformed flux curves are shown labeled by which exponential 1, 2 or 3 from equations (13) or (14) dominates in determining the flux above a certain energy at a certain location. From inspecting Figures 8 to 12 we can arrive at the following general statements:

(I) Near the equator the agreement of the measured fluxes and transformed fluxes is quite good where direct comparison can be made. This agreement remains quite good going off-equator up to a location dependent on-energy and location but given very roughly by $B / B_{O} \sim 3$ For proton energies of a few Mev. For higher energies (Fig. Il and Fig. 12) the region of agreement is quite small. 
(2) For locations well off-equator there is a decided discrepancy between the measured fiuxes and transformed fluxes. The neawred fluxes are consistently higher.

There are at least two ways in which the off-equator fluxes might be wade larger than those giver by the transformation here. First the may be processes which violate either the first or second adiabatic invariant that usualiy will tend to move particles down siela lines. In this way we could populate off-equator locations by ovirg particles from the equatoriai region down field lines. We wust be careful not to do this so eificiently that the equatorial iluxes are changed significantly, otherwise we would lost the agreement that we do have at the equator. But most of the Davis protons in a wube of force are confined quite close to the equator so maybe supplying whe lower altitude population can be accomplished without damaging the equatorial agreement.

A second way of supplying the off-equator proton flux, which seems more likely to me, would involve a second source of protons. It is well estajlished that neutron decay protons produce most of the observed $\mathrm{E}>30 \mathrm{Mev}$ protons for $\mathrm{L}<1.5$. The neutrons decay source extends outwards into the outer belt, falling off about a $R^{-2}$. These protons will also be acted on by the process that violates the third invariant and these protons will also move in I. These will tend to 
aiffuse outwards away from the source. Tverskoy (1964) suggested that unis process might be responsible for the sharp outer edge of the inner belt proton distribution. We have the interesting possibility that outer zone protons diffuse inwards to fill the region near the equator at low $\mathrm{L}$ values and simultaneously inner zone protons are diffusing outwards to fill the higher $B$ parts of field lines as shown in Fig. 13 This is what is expected by analogy to gradient diffusion where particles tend to move away from regions of high flux. This idea has not been tested quantitatively.

Other Particles

Recently experiments have indicated that besides protons, other particles also undergo I diffusion. Frank (1965) has shown a radially inwards moving wave of electrons in the outer belt following a magnetic storm. The wave had a radial velocity

$$
\mathrm{v} \cong k L^{8}
$$

This wave has the right properties to be due to I diffusion concerning $\therefore$ and J. The magnetic pumping process described by Parkor (1960) Davis and Chang (1962), and Nakada and Meaa (1965) will operate equaily well on all particles that have the same drift period $\tau_{D}$ around the earth since this is the oniy particle diameter that enters the theory. Tais means that eiectrons and protons of the same energy will experience 
anilly the same I difîsion. However the electron problem appears wore conjicated than the proton one. Other processes such as precipitation or pitci angle scattering and in general short lifetime seem characteristic of electrons, while for protons I diffusion appears to be dominant. Van Allen (1965) has recently identified $\alpha$-particles in the outer rauiation belt. If some of the protons in the outer belt are due to I diffusion inwards from the magnetopause, then we would expect $\alpha$-particles there too. It is now quite well established that there are several percent $\alpha$-particles in the solar wind. If these get reasonably thermalized in the transition zone, as seems to occur for protons and electrons, then we should have for the fiux of $\alpha$-particles in the transition zone

$$
j_{\alpha}(E) \approx k j_{p}(E) \quad \text { where } k \sim .05
$$

Assuming that these $\alpha$-particies can get through the magnetopause, is seems to be the case for the protons, then by analogy to the known proton energy spectrum inside

$$
j_{p}(E)=k_{p} e^{-E_{p} / E_{o p}}
$$


we woulc expect the $\alpha$-parificle energy spectrum to be

$$
\dot{j}_{\alpha}(E)=k_{\alpha} e^{-E_{0} / E_{0 \alpha}}
$$

Also as a result $0 \hat{i}$ the thermalization we should have

$$
E_{O \alpha}=E_{O p}
$$

$\hat{I} 0:$ all locations in the magnetosphere wizere loss processes are not too importart. Loss processes will be difierent for protons and $\alpha$-particies and provijiy will change the two spectra in different ways. Loss processes seem important for protons for $\mathrm{I}<3$.

This situation where the proton and aipha particle energy spectra are the same should be true as iong as we have steady state, without loss for both particles, that is, where the Fokker Planck equation can be written (Davis and Chang, 1962)

$$
\frac{\partial f}{\partial t}=\frac{\partial}{\partial r}[<\Delta r>f]+\frac{1}{2} \frac{\partial^{2}}{\partial r^{2}}\left[<(\Delta r)^{2}>f\right]=0
$$


The spectra should be the same for steady state because we would expect the diffusion process to operate on the two particles in similar ways so that

$$
\frac{\left\langle\Delta r_{\alpha}\right\rangle}{\left\langle\left(\Delta r_{\alpha}\right)^{2}\right\rangle}=\frac{\left\langle\wedge r_{p}\right\rangle}{\left\langle\left(\Delta r_{p}\right) 2\right\rangle}
$$

which from (20) would give $j_{\alpha}(E)=k j_{p}(E)$. The two sets of particles don't have to diffuse with the same velocity. They can move through eacis otiner and probably will, because protons and $\alpha$-particles of the same energy will have different drift periods

$$
\tau \alpha \frac{I}{v_{D}}=\left[\frac{m c}{z e B R}\left(\frac{v_{l}^{2}}{2}+v_{1:}{ }^{2}\right)\right]^{-1}
$$

From (22) for protons and $\alpha$-particles of the same kinetic energy

$$
\frac{\tau_{\alpha}}{\tau_{p}}=2
$$


Wis means that a different set of magnetic disturbances will cause violation of the $\bar{q}$ invariant for protons and $\alpha$-particles of the same energy.

A prediction of this model of $L$ difiusion is that inside the magnetosphere the proton and $\alpha$-particle energy spectra will be related by

$$
\frac{j_{\alpha}(E)}{j_{p}(E)}=\frac{k_{\alpha} e^{-E / E_{0}}}{k_{p} e^{-E / E_{O}}}=k \sim .05
$$

in the region of $I$ space where loss is unimportant (roughly I > 3 ). Also if the suggestion put Iorward here is correct, that outer belt protons at large $B / B_{0}$, i.e. well of $f$ the equator, are drifting outwards from the inner belt, then we would not expect $\alpha$-particles to exist in this region of space. If $\alpha$-particles are found at large $B / \bar{B}_{0}$, then maybe violation of the $\mu$ or $\mathrm{J}$ invariants is important for outer belt protons. 
REFERENCES

Davis, I. R., R. A. Horfman, J. M. Williamson, "Observations of Protons above 2 Earth Radii", Trans. A.G.U., 45, 84, 1964.

Davis, L. R. and J. M. Williamson, "Low Energy Trapped Protons", Space Research III, $0.365,1963$.

Davis, L., and D. 3. Chang, "On the EIfect of Geomagnetic Fluctuations on Trapped Particles", J. Geophys. Res., 67, 2169-2179, 1962.

Kellogg, P. J., "Van Allen Radiation of Solar Origin", Nature, 183, 1295-1297, 1959.

Furiker, E. N., "Geomagnetic Fluctuations and the Form of the Outer Zone of the Van Allen Radiation Belt", J. Geophys. Res., 65, 3117-3130, 1960.

Nakada, M. P., J. W. Dungey and W. N. Hess, "On the Origin of OuterBelt Protons, 1, I. Geophys. Res., 70, 3529-3533, 1965.

Tverskoy, B. A., "Dynamics of the Radiation Belts of the Earth II", Geomagnetism and Aeronomy, 3, 351-366, 1964.

Frank, I. A., "Inward Radial Diffusion of Electrons of Greater than 1.6 Miliion Electron Volts in the Outer Radiation Zone", J. Geophys. Res., 70, 3533-3541, 1965.

Nakada, M. P., and G. D. Mead, "Diffusion of Protons in the Outer Radiation Belt", I. Geophys. Res., to be published.

Van Allen, J. A., and S. M. Krimigis, "Trapped Alpha Particles in the Earth's Outer Radiation Zone", Trans. A.G.U., 46, 140, 1965. 
Fillius, R. W., and C. E. McIlwain, A survey of inner zone protons, University of California (at San Diego) paper, April 13, 1965. Fililus, R. W., and C. E. McIlwain, The anomalous energy spectrum of protons in the earth's radiation belt, Phys. Rev. Letters, 12 , 609-612, June 1, 1964 . 


\section{FIGURE CAPIIONS}

i. Lor energy proton spectra at various distances from the earth, fro: Davis and Williamson, (1963)

¿. Variation of equatorial pitch angle with $I$ for motion conserving i. axi j.

3 . The ratio of proton energies at $L$ to that at $L=7$, assuming $\mu$ and J constant, for various equatoriai pitch angles at $I=7$.

4. Variation of $E_{0}$ with $I$ for various equatorial pitch angles.

5. The velocity distribution function $f$ vs. I for various values of $\mu$ and $J$ determined from Davis data. The curves for different it and i have been normalized in the Region $L \sim 4$.

7 The exponential fit to the energy spectrum of Davis, Hoffman and iiliamson, 1964 .

Z. A cumparison of the transformed Davis, Hoffman and Williamson ciata wit: the spectrum measured by Fillius and McIlwain at $I=2.4$.

8. A cutisarison of transformed Davis, Fofiman and Williamson,( 1964 ) iate with measurements by Fillius and McIlwain of protons of i.i to 14 MeV. The curves are identified by which exponentia: terii from Eq. 13 or 14 dominates in the transformed data.

9. A comparison of transformed Davis, Hoffman and Williamson, (1964) data with measurements by Fillius and McIlwain of protons of 1.6 to $7.1 \mathrm{MeV}$. The curves are identified by which exponential term from Eq. 13 or 14 dominates in the transformed data. 
ic. A cunpurtion of traneformer Davis, Thrmen and Williamson, (icin) data with measurements by filitus and reilwain of protons of 2.25 to $4.7 \mathrm{MeV}$. The curves are identified by which exponentiai ter: from Iq. 13 or 14 dominates in the tranjformed data.

11. A cuparison of transformed Davis, IJfiman and Williamson, (19ut) data with measurements by Fillius ari Xcilwain of protons of 18.2 to $25 \mathrm{MeV}$. The curves are idchuficd by which exponential tem: irom Eq. 13 or $1 /$ doninates in the transformed data.

12. A cumparison of transformed Davis, Norman and Williamson, igh,t, dativ with measurements by Filzius and McIlwain of protons of 35 to $63 \mathrm{MeV}$. The curves are identiried by which exponentia: ter... From Eq. 13 or 14 doninates in the transformed data.

15. A posible madel to explain of -equatorial low energy protons. Protons travei in from the magretopaise near the equator and travel outward from the inner radiation zone off-equator. 


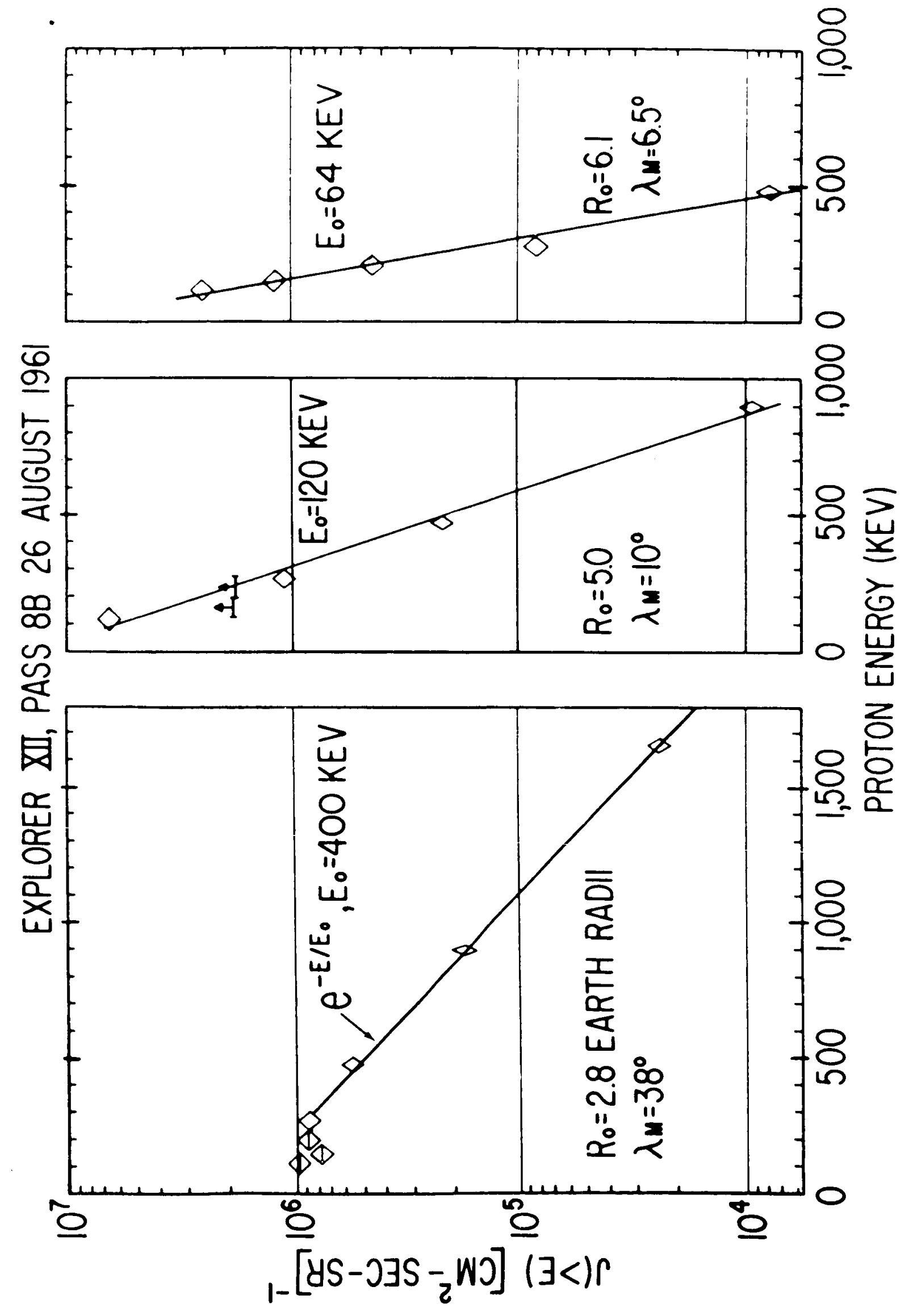




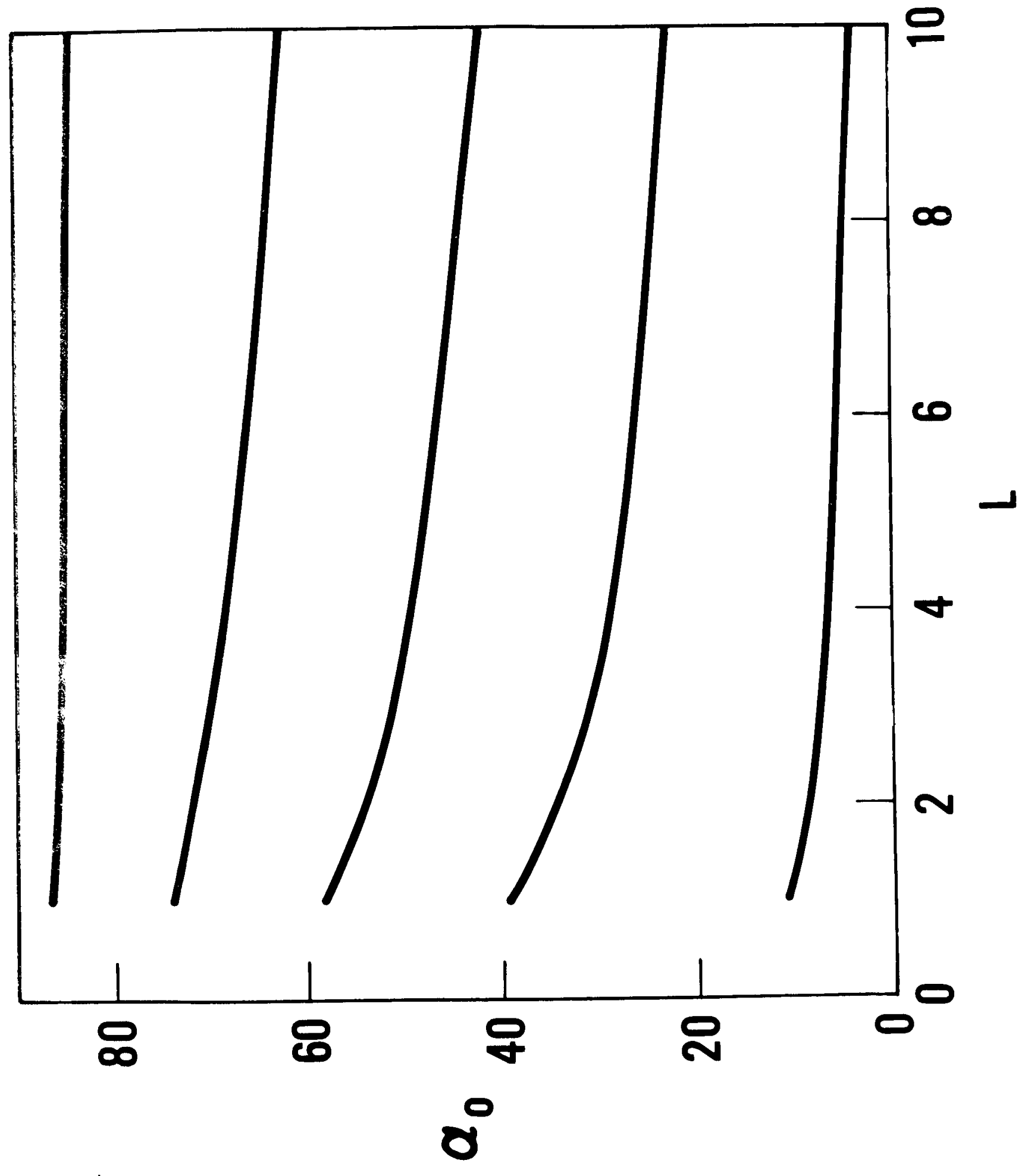




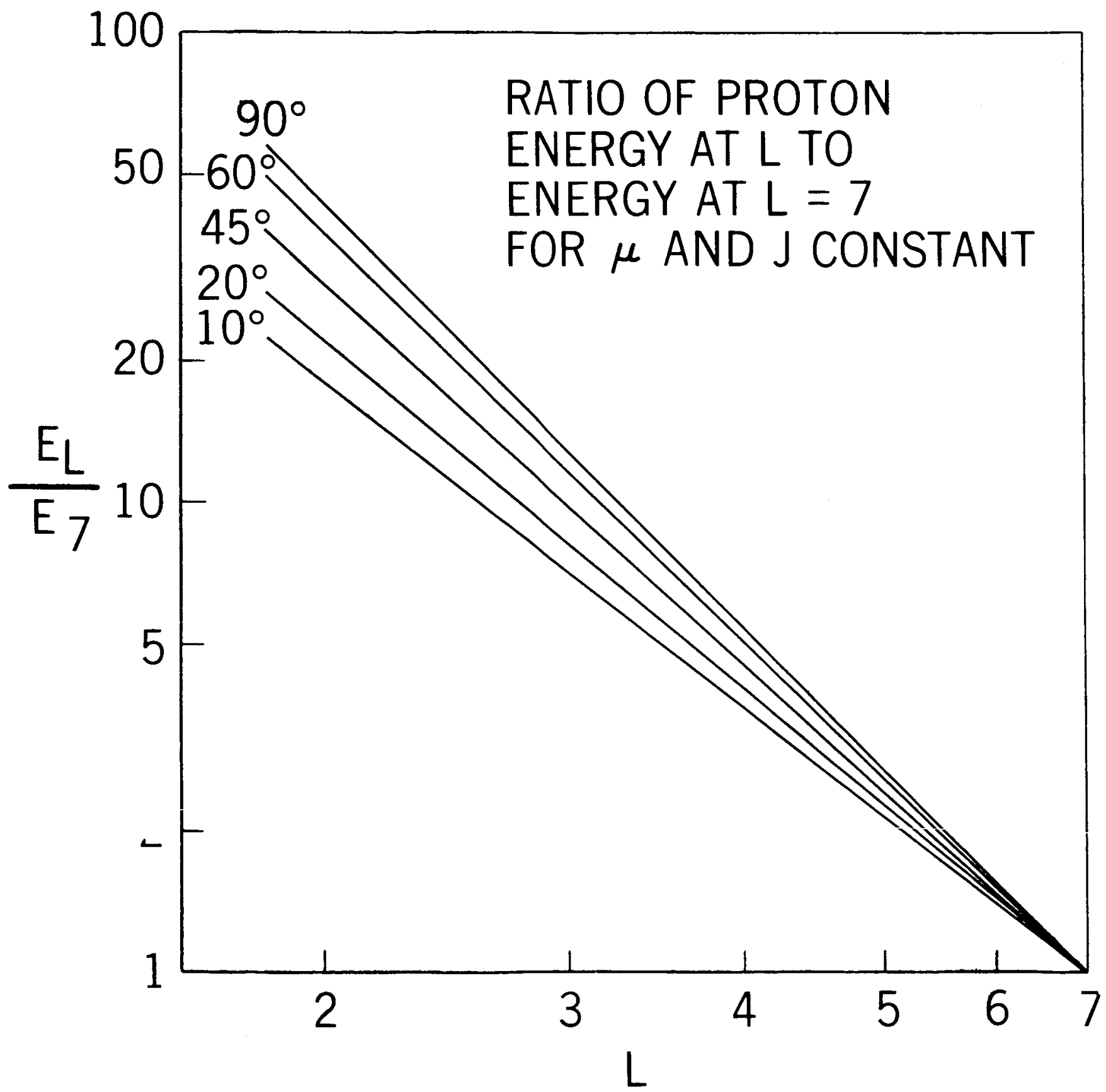




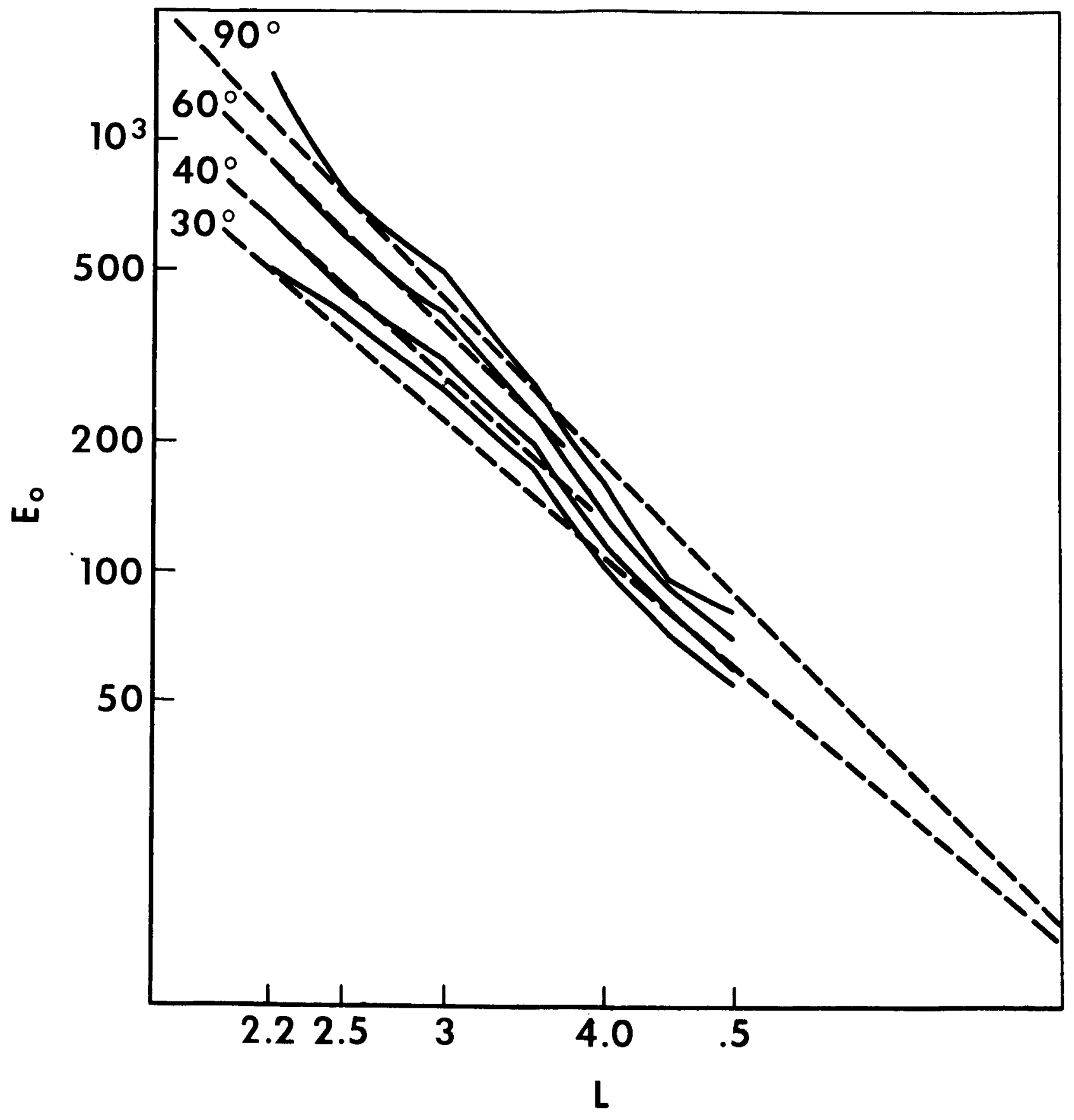




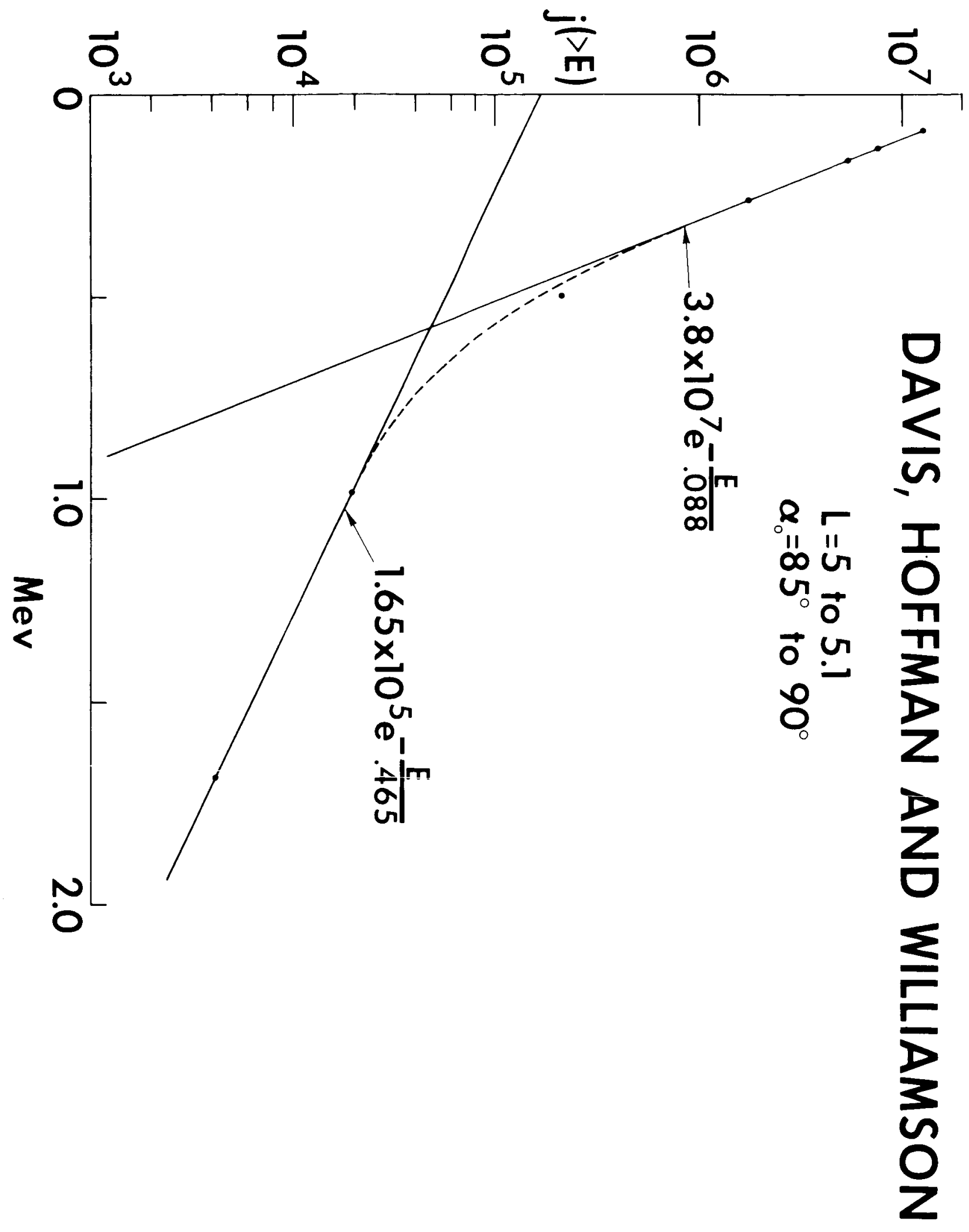




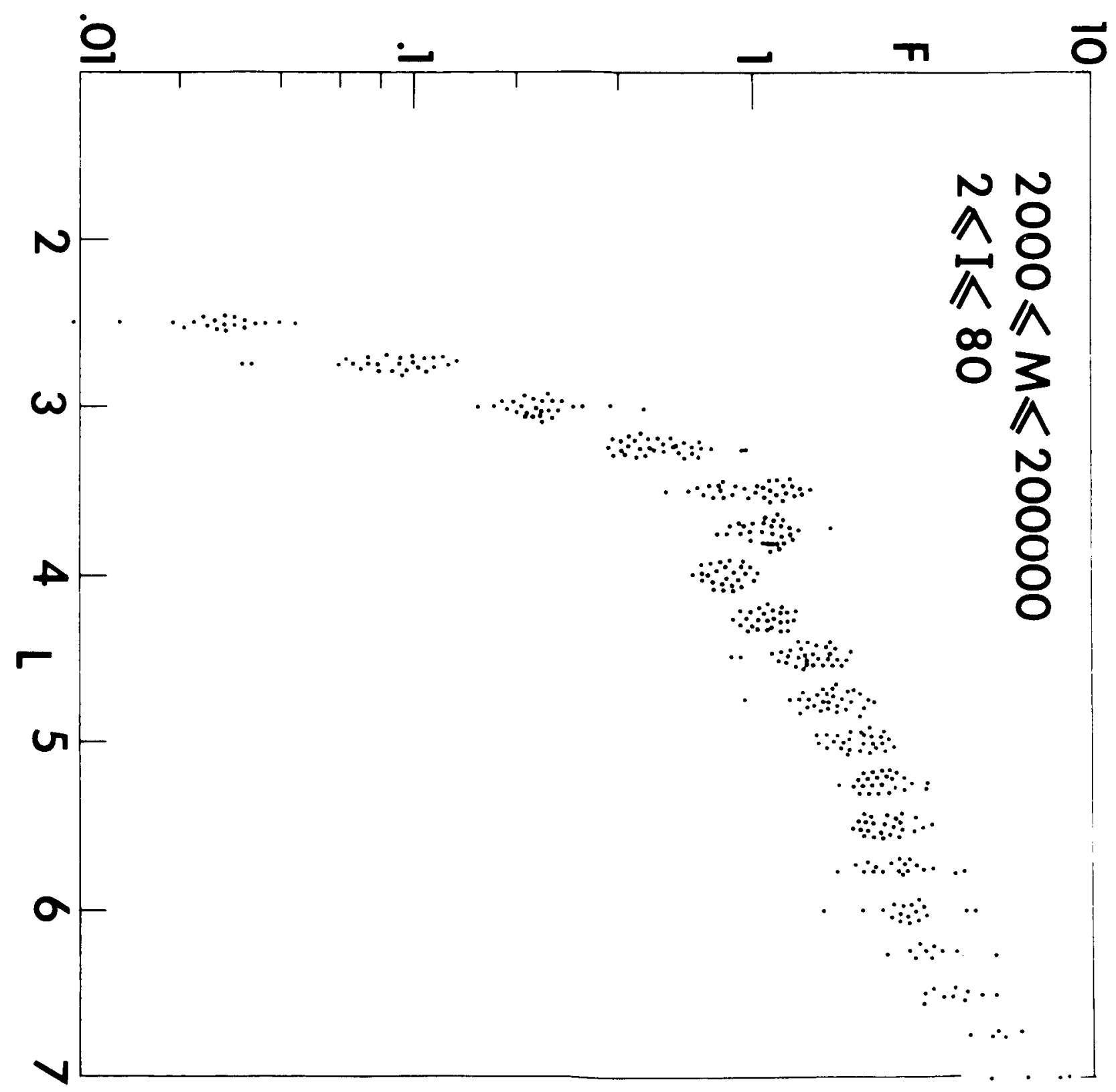




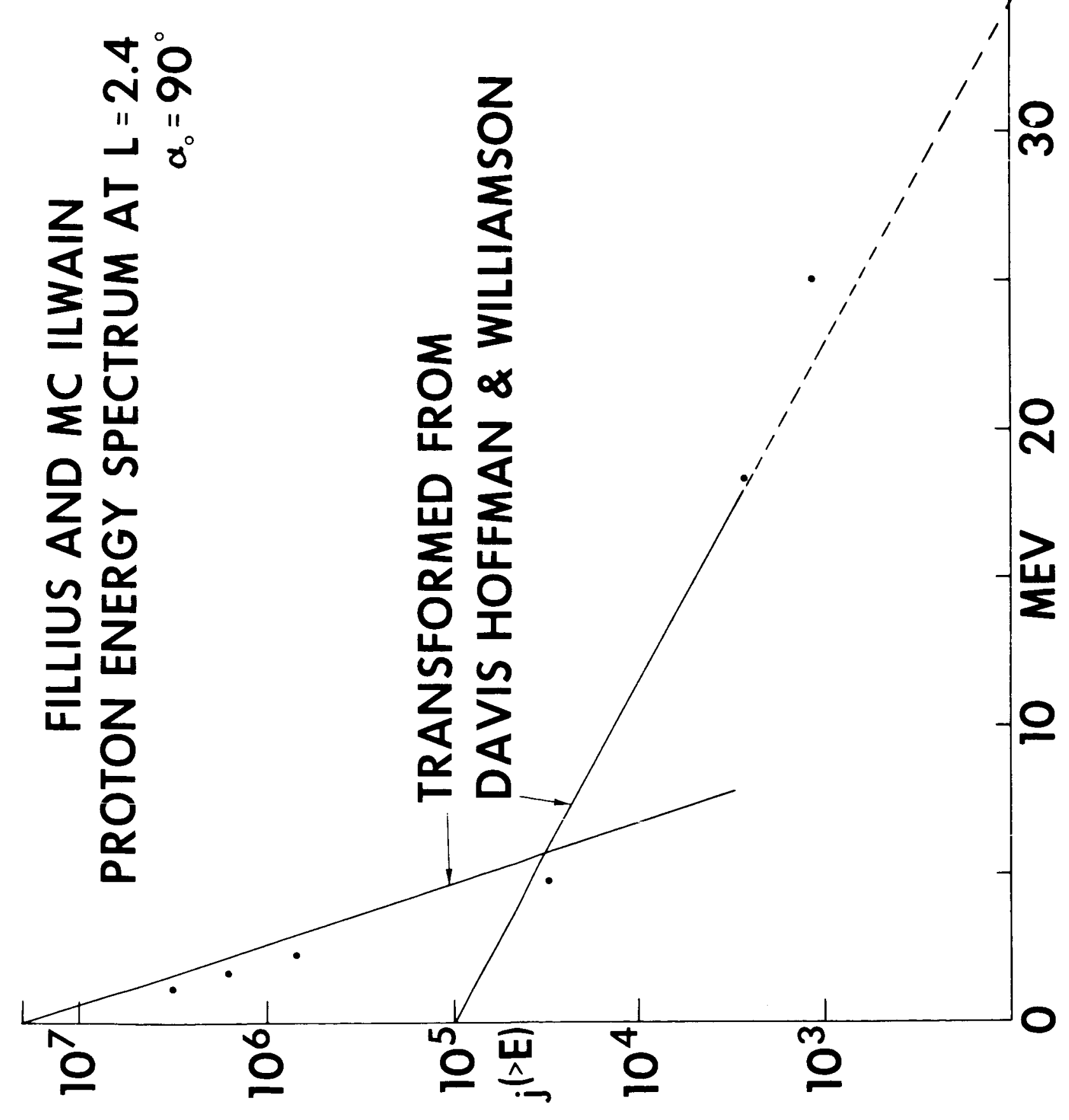




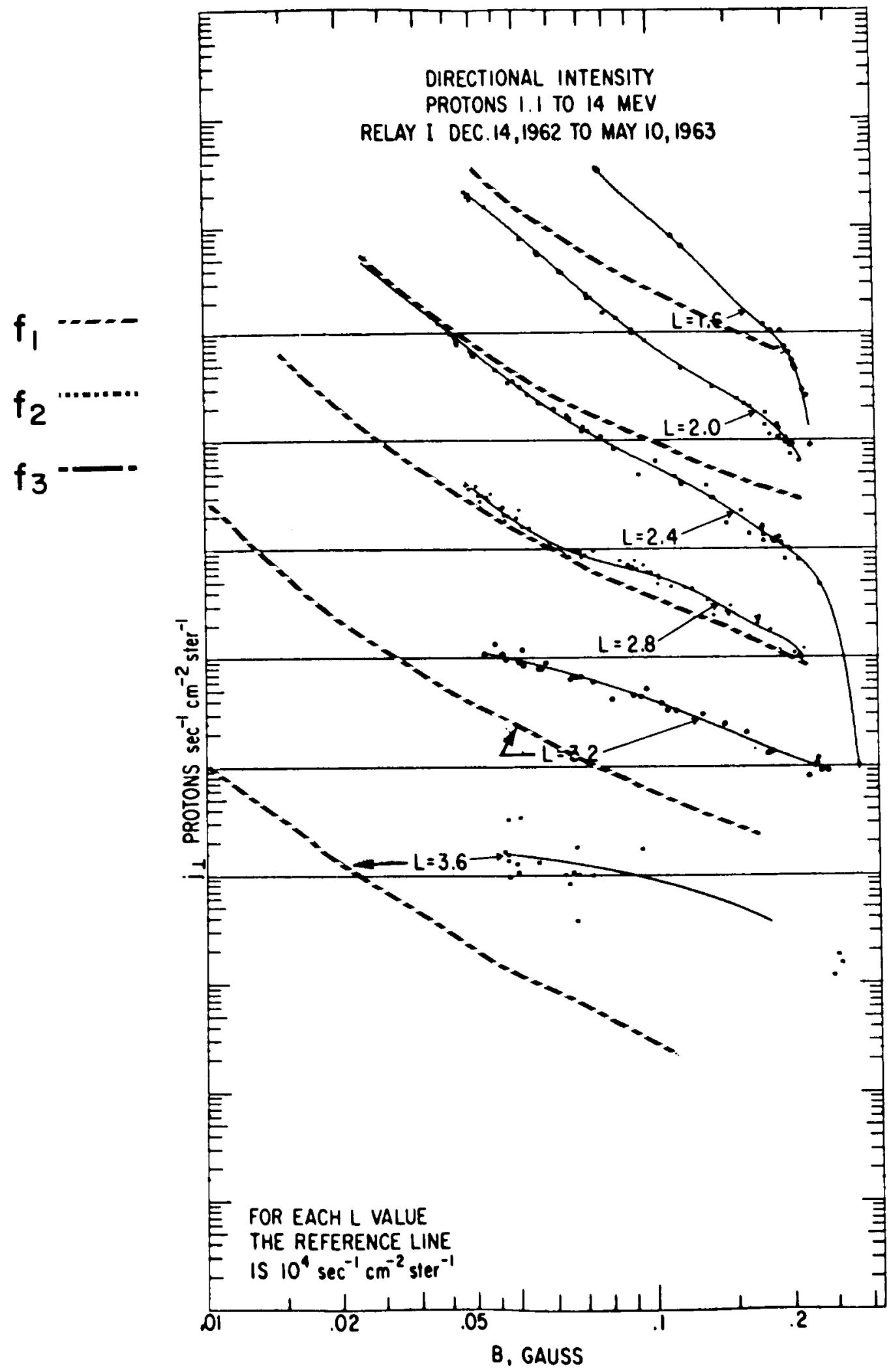




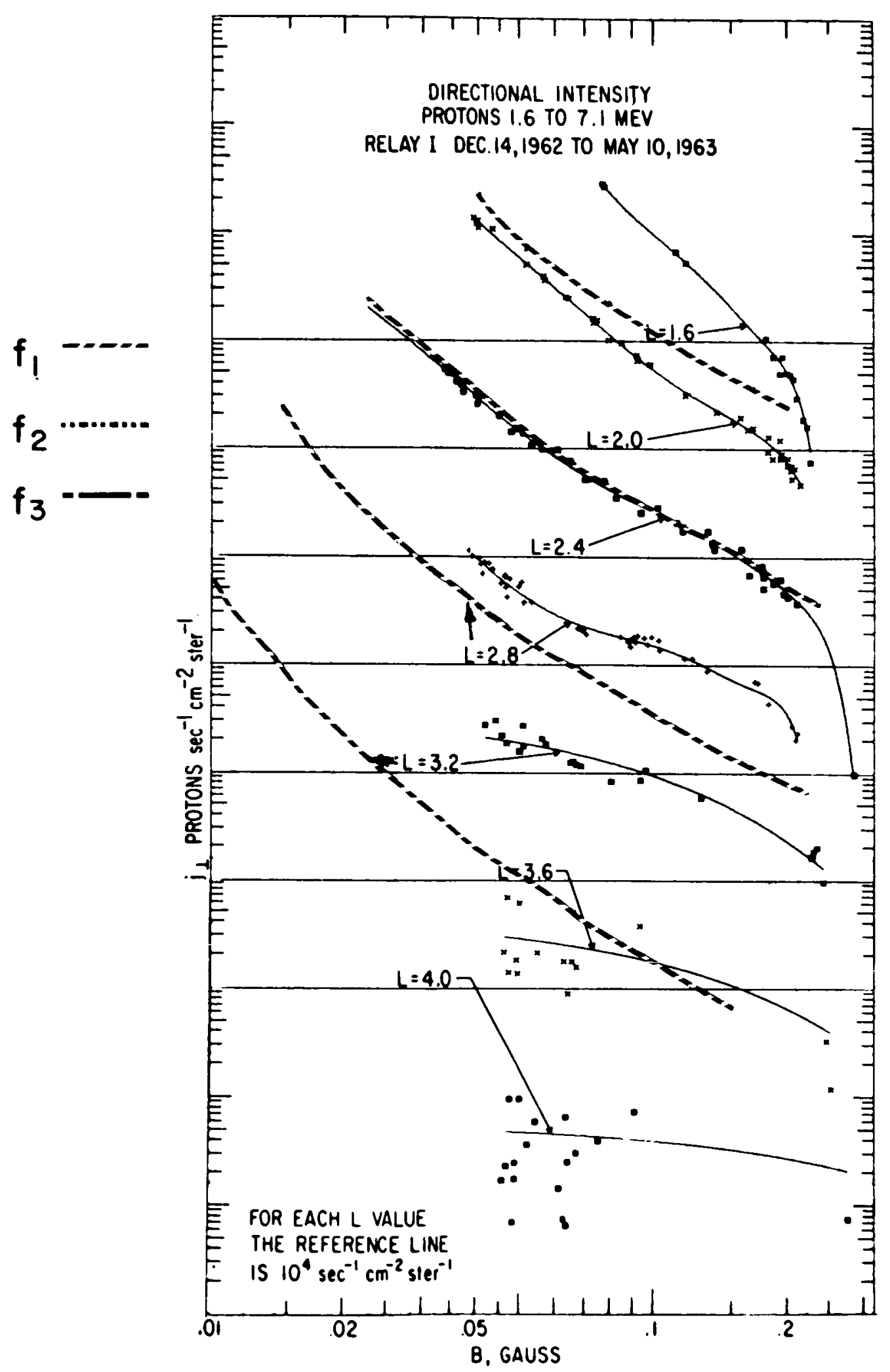




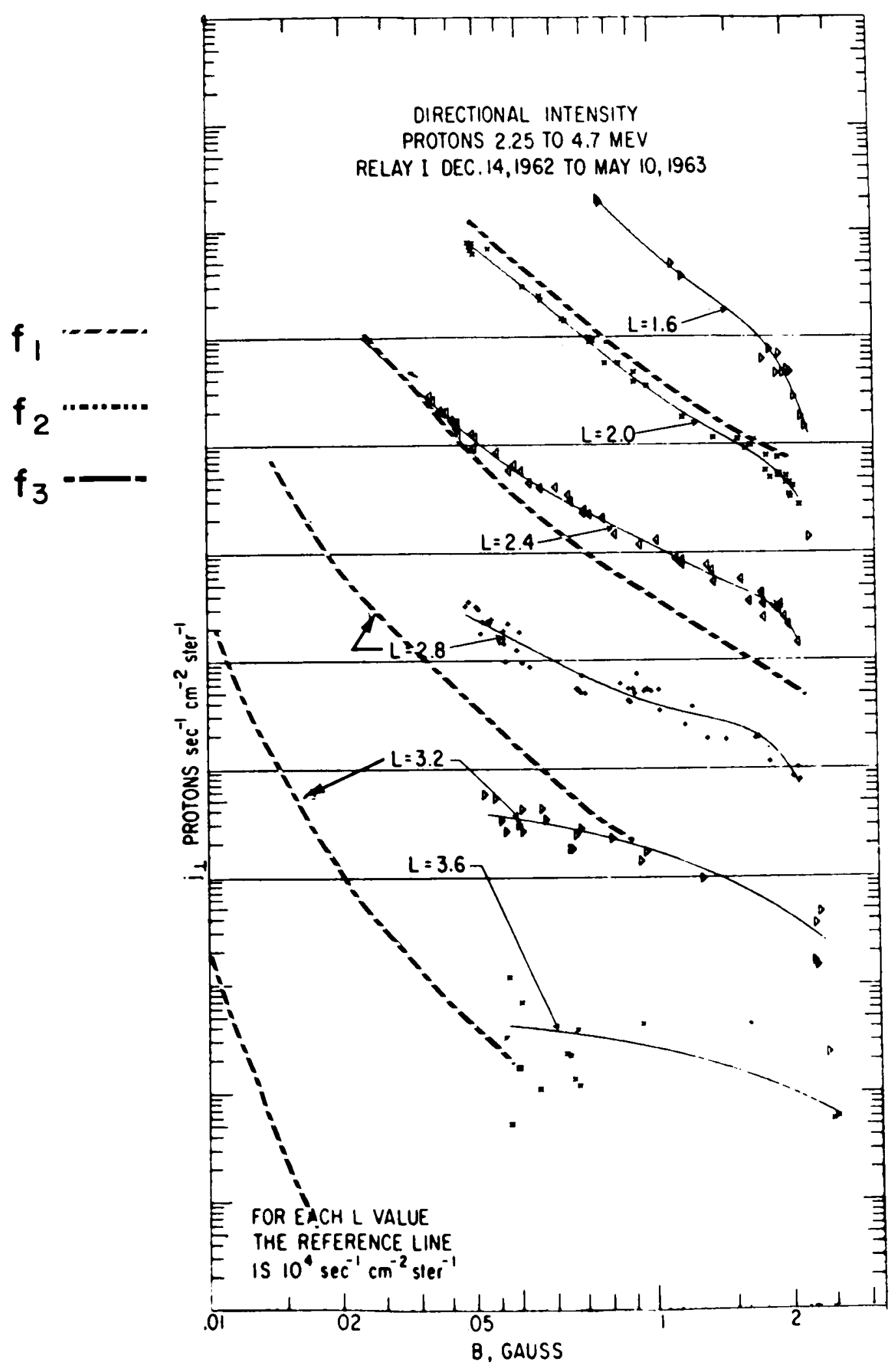




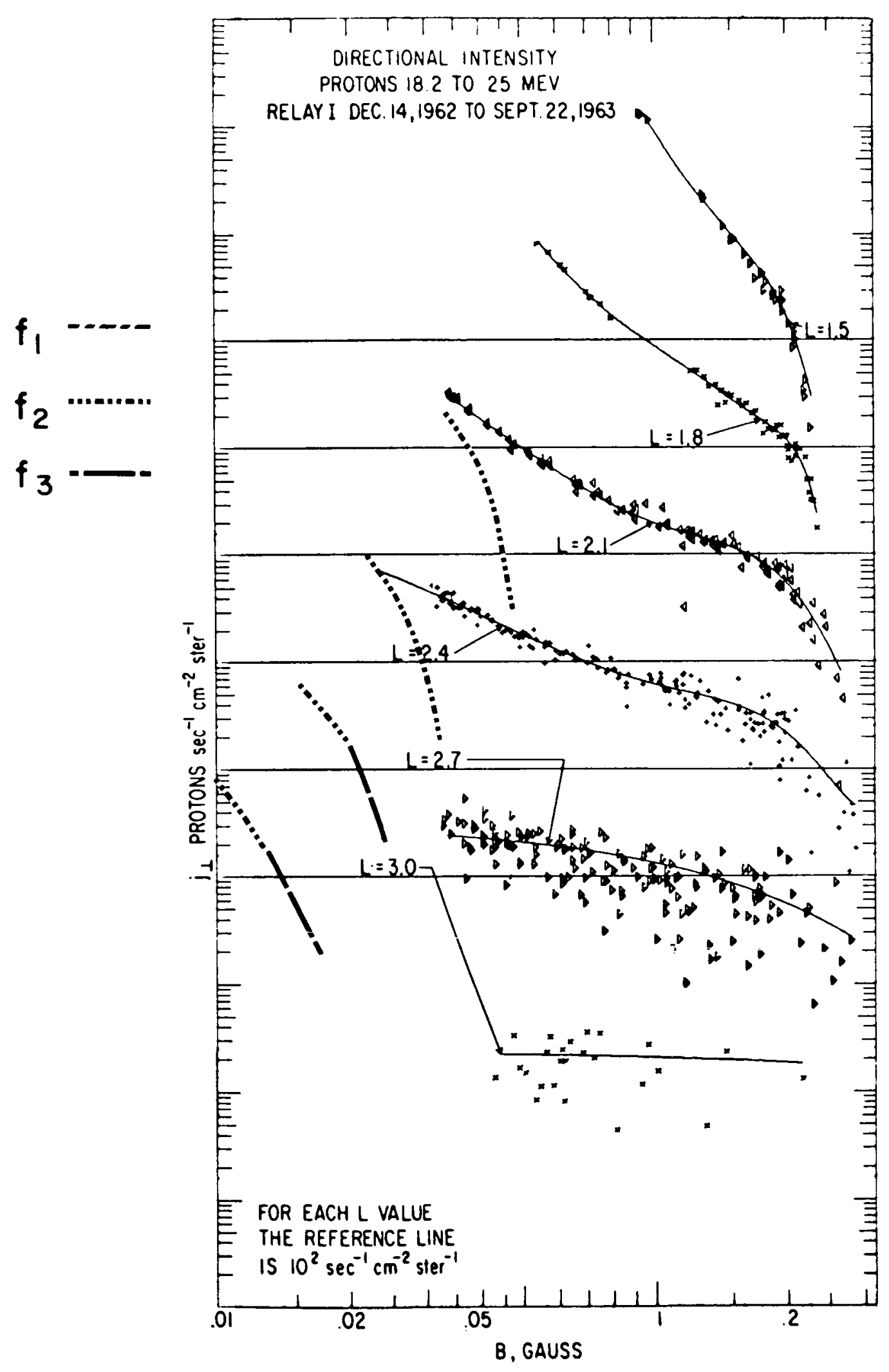




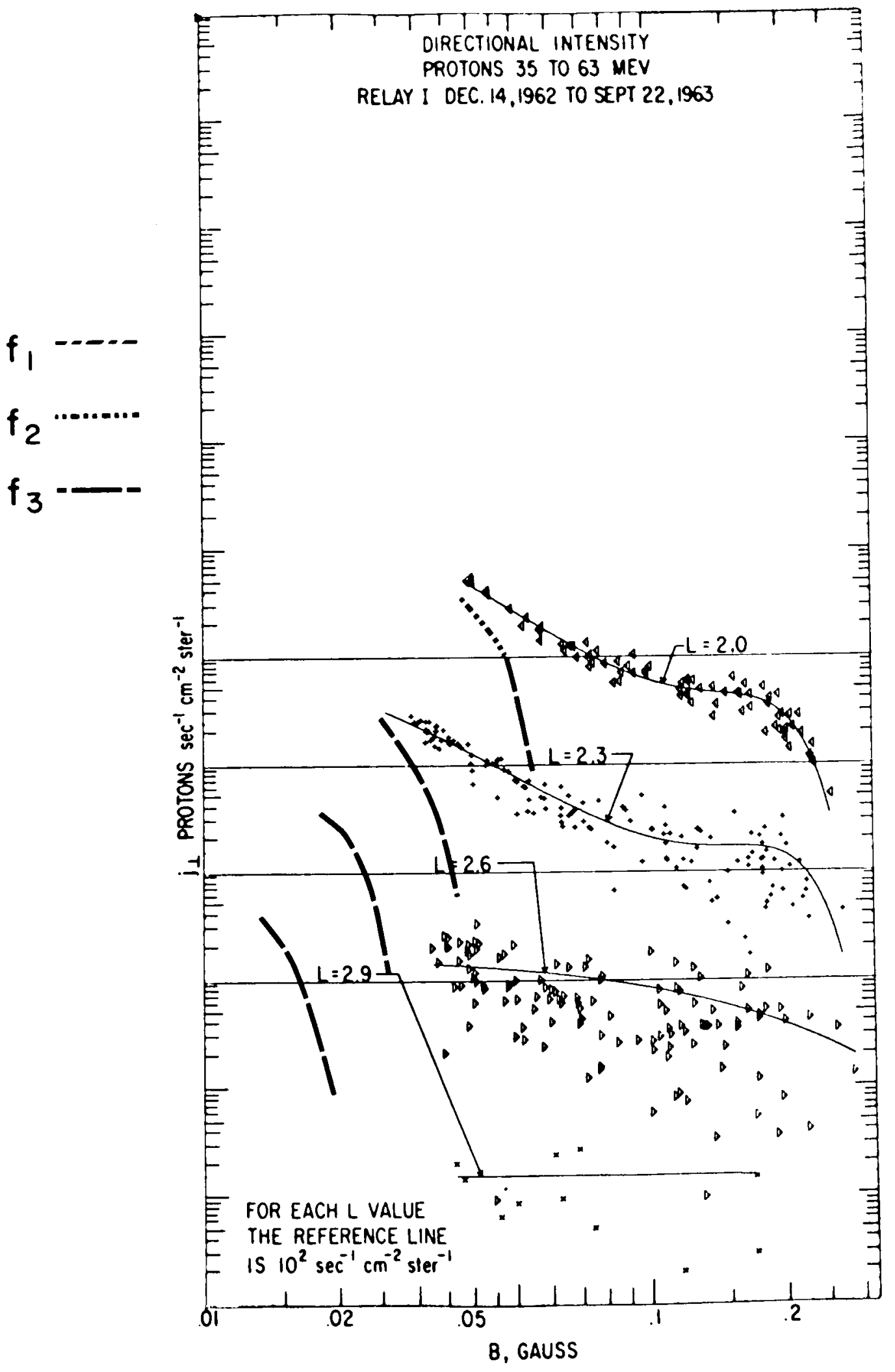


MAGNEIOPAUSE

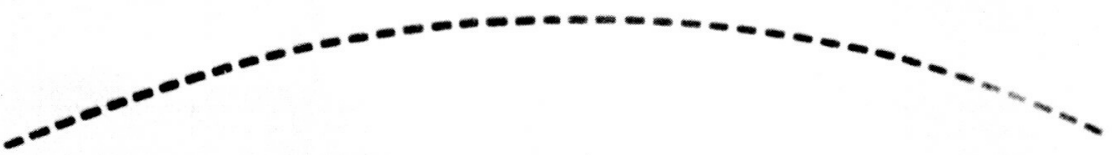

